\title{
Las acciones de modificación en una muestra de cuentos quechua del sur del Pastaza
}

\section{The actions of modification in a sample of quechua tales of southern Pastaza}

\author{
Alejandra Antoinette Dioses Tarazona \\ Universidad Nacional Mayor de San Marcos, Lima-Perú \\ alejandra.dioses@gmail.com \\ ORCID https://orcid.org/oooo-0001-5612-0288
}

\begin{abstract}
Resumen
En este trabajo se analizan oraciones de una muestra de cuentos recopilados de la variedad quechua del sur del Pastaza bajo la perspectiva de la semántica formal. El estudio se centra en el análisis de las expresiones, que se encuentran de manera explícita en los textos, donde se aprecian acciones de modificación. El objetivo de este trabajo es identificar los elementos de la acción en cada oración: agente, paciente y propiedad atributiva. Para ello, las oraciones seleccionadas serán formalizadas. Además, se busca proponer una estructura sintáctica del predicado donde se puedan identificar los roles mediante la teoría X-barra y se caracterizarán los participantes dentro de cada oración mediante los sufijos añadidos que estos posean.
\end{abstract}

Palabras clave: Quechua del sur del Pastaza; semántica formal; modificación; teoría X-barra

\begin{abstract}
This work analyzes sentences in a sample of collected stories from the variety of Southern Pastaza Quechua under the perspective of formal semantics. This study is focused on the analysis of expressions, that are found explicitly in the text, where modification actions are appreciated. The objective of this work is to identify the elements of the actions in each sentence: agent, patient and attributive property. For that reason, the selected sentences will be formalized. Furthermore, it seeks to propose a syntactic structure of the predicate where the elements of the actions can be identified through the X-bar theory and they will be characterized in every sentence through the added suffixes that they possess.
\end{abstract}

Keywords: Southern Pastaza Quechua, formal semantics; modification; X-bar theory

\section{Resumo}

Este artigo analisa frases de uma amostra de histórias recolhidas da variedade Quechua do sul de Pastaza, a partir da perspectiva da semântica formal. O estudo centra-se na análise das expressões, que se encontram explicitamente nos textos, onde as acções de modificação podem ser apreciadas. O objectivo deste trabalho é identificar os elementos de acção em cada frase: agente, paciente e propriedade atributiva. Para este efeito, as frases seleccionadas serão formalizadas. Além disso, o objectivo é propor uma estrutura sintáctica do predicado onde os papéis podem ser identificados 
Las acciones de modificación en una muestra de cuentos quechua del sur del Pastaza Alejandra Antoinette Dioses Tarazona

através da teoria da barra $\mathrm{X}$ e os participantes dentro de cada frase serão caracterizados por meio dos sufixos adicionados a eles.

Palavras-chave: Quechua Pastaza do Sul; semântica formal; modificação; teoria das barras X

\section{Introducción}

La familia lingüística quechua es amplia y posee múltiples variedades que se hablan en distintos países sudamericanos, entre los cuales está Perú. Una de estas variedades es la que se habla al sur del Pastaza, en la región norte de la amazonía. Como ya es sabido, el quechua es una lengua aglutinante que posee una amplia diversidad de sufijos que cumplen diversas funciones. Ante ello, surge la interrogante de la existencia de sufijos específicos que denoten determinados roles semánticos, en este caso, los involucrados en una acción de modificación. Por esa razón, bajo los estudios de la Semántica formal, se realiza un análisis de quince enunciados para determinar cuáles son los sufijos que indican los papeles de agente, paciente y propiedad atributiva dentro de las acciones de modificación. Las oraciones fueron extraídas de la compilación de relatos denominada Cuentos folklóricos de los quechua del Pastaza, a la cual se tuvo acceso virtualmente durante el contexto de la pandemia originada por la Covid-19.

\section{Marco conceptual y teórico}

\subsection{Quechua del sur del Pastaza}

La variedad quechua del sur del Pastaza, también conocida como Inga, pertenece al grupo II-B según la clasificación de Alfredo Torero. Esta variedad abarca comunidades ubicadas a orillas del río Pastaza en Loreto. Tiene una similitud con el quichua que se habla al sur de Ecuador (quichua del norte del Pastaza) pero no son iguales. Se extendió ante un intento de quechuización a las comunidades cercanas al río Pastaza. Acerca del origen del quechua en la zona del sur del Pastaza, José Vilcapoma (2016) menciona lo siguiente:

Se dice de tácito acuerdo que los Misioneros del Napo y del Marañón introdujeron en aquellas montañas verdes la lengua quichua, llamada del Inca, o Inga. No obstante, en aquella zona ya existía como elemento de comunicación el quechua, el cual había asentado en esos lugares por presencia de los Incas,

114 Lengua y Sociedad. Revista de Lingüística Teórica y Aplicada 
y que ahora venía con los curas después del III Concilio Limense (1583-1584), obra de adoctrinamiento religioso en quecha[sic] más grande de la colonia. Bajo la dirección de Toribio Alonso de Mogrovejo se impulsa la publicación de catecismos y diccionarios quechua, para el proceso de evangelización. [...] El idioma entre ellos variaba según su procedencia; pero se llegó a imponer el de los Incas, el quíchua, que fue un elemento de cohesión entre todos los moradores de la Región (Costales, 1983:14). El Pastaza, que entonces tenía como nombre río Corino, fue el centro de penetración desde Ecuador. (p. 292)

De acuerdo con Ethnologue 2 , esta variedad quechua tenía 1550 hablantes en el año 2000, de los cuales 310 de ellos eran monolingües. También señala que la lengua es usada por todos en casa, en el trabajo, en la iglesia y durante los juegos, aunque algunos también utilizan el español. Tiene la estructura tipológica de sov, la misma que posee la familia quechua como macrolengua según la categorización del ILV. Además, esta lengua se encuentra en etapa 5, es decir, está en desarrollo, hay un buen uso de esta y tiene una forma estandarizada de escritura; pero, a pesar de ello, no está muy extendida ni es totalmente sustentable.

\subsection{Semántica formal}

Para este trabajo, se utilizará el lenguaje formal ya que facilita el tipo de análisis de los enunciados seleccionados debido a que se revisarán estructuras sintácticas. En cuanto al lenguaje formal, Leticia Santos (2017) menciona:

El lenguaje formal es un lenguaje artificialmente creado como herramienta de análisis científico en el que representar ciertos contenidos para estudiar sus características. La utilidad de estos lenguajes se encuentra en su estructura sintáctica. Los lenguajes formales como sistemas lógicos son fundamentalmente sintácticos de forma que abstraen los contenidos de los enunciados. Esto posibilita representar en un mismo lenguaje distintas realidades con la garantía de que tales sistemas preservan la verdad pues son estructuras donde se garantiza la inferencia válida. Lo que importa en un sistema lógico es si las fórmulas que se deducen del sistema son verdaderas. Esto se consigue regimentando la sintaxis a través de definiciones recursivas de los elementos y de las relaciones (siempre deductivas) del sistema de modo que haya transparencia sintáctica. De esta forma, los símbolos del lenguaje así como la forma

Cita textual copiada literalmente. [quecha] [quechua]

Información extraída de la página web de Ethnologue. 
Las acciones de modificación en una muestra de cuentos quechua del sur del Pastaza Alejandra Antoinette Dioses Tarazona

en que pueden combinarse para crear símbolos complejos y para justificar la inferencia de unos a otros están especificados. (p. 158)

La Semántica formal estudia las relaciones entre los elementos sintácticos de un enunciado, centrándose en el análisis de la forma y no del contenido (significado). Mediante esta perspectiva, se buscará proponer una estructura que represente las acciones desarrolladas en determinados enunciados tomados de una muestra de cuentos quechua del sur del Pastaza. Respecto a la Semántica formal, Germán Guerrero (2010) explica lo siguiente:

La distinción entre sintaxis y semántica se puede trazar en los siguientes términos. Si se parte de considerar un lenguaje como un conjunto de expresiones, como es usual en estos estudios, las propiedades sintácticas de una expresión de un lenguaje sólo tienen que ver con sus relaciones con otras expresiones, dejando de lado cualquier consideración sobre el significado o la interpretación de las mismas. Por la otra parte, una propiedad semántica de una expresión concierne a la relación de la expresión con el mundo, con el universo de discurso. La semántica estudia el significado de las palabras y la verdad o falsedad de los enunciados. Estas relaciones semánticas, gracias a los trabajos de Tarski, pueden expresarse en términos de lo que se llama interpretación o modelo de un lenguaje formal, de modo que la noción de modelo hace parte de la semántica. (p. 172)

Para complementar lo mencionado sobre la Semántica formal, se añade lo señalado por Muñoz (2006), quien indica lo siguiente:

Efectivamente toda oración remite a un hecho si el hecho ocurre, en cuyo caso decimos que esa oración es verdadera; si no ocurre, decimos de ella que es falsa. La verdad entonces es una propiedad de las oraciones que refieren, remiten o representan hechos en el mundo. Naturalmente cada oración tiene o puede tener un significado distinto, pero las referencias posibles de las oraciones son sólo dos: lo verdadero o lo falso (al menos en una semántica bivalente). La verdad es, en consecuencia, una noción semántica por medio de la cual valoramos o juzgamos la relación que se establece entre las oraciones que expresan hechos del mundo y estos mismos hechos. (p. 2)

116 Lengua y Sociedad. Revista de Lingüística Teórica y Aplicada 
Las acciones de modificación en una muestra de cuentos quechua del sur del Pastaza

Alejandra Antoinette Dioses Tarazona

\subsubsection{Teoría de Modelos}

Leticia Santos (2017) expresa que el lenguaje debe contar tanto con una estructura sintáctica como semántica. Para el caso del lenguaje formal, se emplea la Teoría de modelos para interpretar su semántica, partiendo de la noción matemática de la Teoría de conjuntos. De esta manera, la autora establece que:

[...] en la Teoría de modelos se opta por una definición semántica extensional: se asume que los significados de los símbolos son sus denotaciones. La tarea semántica entonces consiste en interpretar un lenguaje (asignar denotaciones a los símbolos del lenguaje): primeramente se deben asignar denotaciones a los elementos básicos y posteriormente explicar cómo a partir de la combinación de estos se obtienen los significados de las fórmulas complejas. (p. 158)

Asimismo, Portner admite que, en la Teoría de modelos, «los términos (constantes y variables) denotan individuos, los predicados denotan conjuntos de individuos y las oraciones valores de verdad. La Semántica formal se construye en definitiva a partir fundamentalmente de denotaciones, como valores semánticos, y funciones» (2005, en Santos, 2017). Habiendo mencionado lo anterior, se agrega lo desarrollado por Muñoz (2006), quien se explica de la siguiente manera:

Desde esta perspectiva, el lenguaje puede comprenderse como un sistema de reglas de producción que generaba cadenas de símbolos como meras estructuras sintácticas a las que se les podía asignar interpretaciones rígidas para que refirieran a elementos del mundo ya fuera el mundo natural o cualquier mundo formal, por un lado. [...] Además, en la medida en que toda oración o expresaba un hecho del mundo o no lo expresaba, es decir era verdadera o falsa, podíamos articular toda una semántica veritativo-funcional sobre dos principios fundamentales que nos permiten comprender las condiciones en las que las oraciones son o verdaderas o falsas. (pp. 2-3)

\subsubsection{Las acciones}

Las acciones según las define Moreno Cabrera (2003) son «[...] relaciones entre procesos y entidades. Estas relaciones se interpretan habitualmente como relaciones de agentividad o causatividad: una entidad origina, controla o es responsable de que se produzca un proceso determinado» (p. 165). Además, indica que el concepto de proceso es empleado como base para poder definir las acciones. Estas relaciones serían denotadas mediante el aleph (א) y se expresan mediante las siguientes estructuras: 
a. $\aleph\left(\Upsilon_{2}\left[\mathrm{P}\left[\Sigma\left(\Upsilon_{1}, \Phi\right) \rightarrow \sum\left(\Upsilon_{1}, \Theta\right)\right]\right]\right)$

b. $\quad \aleph\left(\Upsilon_{2}\left[\sum\left(\Upsilon_{1}, \Phi\right) \rightarrow \sum\left(\Upsilon_{1}, \Theta\right)\right]\right)$

De esta manera, se puede entender que existen tantos tipos de acciones de acuerdo al tipo de proceso que ocurre, ya que son estos los que las definen. Respecto a esto, Moreno (2003) menciona lo siguiente:

Si el proceso es de desplazamiento, entonces la acción correspondiente se denomina acción locomotiva o locomoción. Por ejemplo, al desplazamiento el coche entra en el garaje le corresponde la locomoción Juan mete el coche en el garaje. [...] Si el proceso es de mutación, entonces la acción correspondiente se denomina acción modificativa o modificación. Por ejemplo. Como el pavo engorda es una mutación, la acción que origina o controla ese proceso, el granjero engorda al pavo es una acción modificativa o modificación. (p. 165)

Para resumir lo mencionado, Moreno plantea el siguiente cuadro clasificando los tipos de acciones de acuerdo al tipo de proceso que las caracteriza:

Tabla 1. Tipos de acciones según el tipo de proceso.

\begin{tabular}{|l|l|}
\hline Tipo de proceso & Tipo de acción \\
\hline Desplazamiento & locomoción \\
\hline Mutación & modificación \\
\hline
\end{tabular}

Nota: Tomado de Moreno, 2003, p. 166.

\subsubsection{Las modificaciones}

Las modificaciones, según Moreno Cabrera (2003), son «[...] acciones en las que se origina o controla una mutación» (p. 168). Estas modificaciones son un tipo de acción que representa un proceso de mutación donde el agente es quien realiza el proceso sobre otra entidad, quien será el paciente, para que cambie de un estado atributivo a otro, es decir, que modifica la propiedad. Él también indica que habrá tantas clases de modificación, así como las hay para las mutaciones. Para ello, presenta un cuadro similar al siguiente:

118 Lengua y Sociedad. Revista de Lingüística Teórica y Aplicada 
Tabla 2. Tipos de modificaciones según las mutaciones.

\begin{tabular}{l|l}
\hline Tipo de mutación & Tipo de modificación \\
\hline Adquisiciones & dotaciones \\
\hline Privaciones & detracciones \\
\hline Aumentos & ampliaciones \\
\hline Disminuciones & reducciones \\
\hline Transformaciones & alteraciones \\
\hline Mantenimientos & preservaciones \\
\hline
\end{tabular}

Nota: Adaptado de Moreno, 2003, p. 169.

A continuación, se presentarán algunos ejemplos con sus respectivas formalizaciones para esclarecer estos conceptos.

(1) Dotación - El sacerdote casó a María.

$\aleph$ (el sacerdote, $\left[\neg \sum\right.$ (María, casada) $\rightarrow \sum$ (María, casada) $\left.]\right)$

(2) Detracción - El fiscal divorció a María.

$\aleph$ (el fiscal, [ $\Sigma$ (María, casada) $\rightarrow \neg \Sigma$ (María, casada)])

(3) Ampliación - Pedro ha perfeccionado sus técnicas de aprendizaje.

$\aleph$ (Pedro, [ $\Sigma$ (técnicas de aprendizaje, buenas) $\rightarrow \sum$ (técnicas de aprendizaje, buenas+)])

(4) Reducción - Jorge ha debilitado sus golpes.

$\aleph$ (Jorge, [ $\Sigma$ (golpes, fuertes) $\rightarrow \Sigma$ (golpes, fuertes $\left.\left.{ }^{-}\right)\right]$)

(5) Alteración - El calor derritió el hielo.

$\aleph$ (el calor, $[\Sigma$ (agua, sólido) $\rightarrow \Sigma$ (agua, líquido) $])$

(6) Preservación - La crisis sanitaria mantiene a los peruanos encerrados.

$\aleph$ (la crisis sanitaria, [ $\Sigma$ (los peruanos, encerrados) $\rightarrow \sum$ (los peruanos, encerrados)])

\subsection{El modelo sintáctico X-barra}

La teoría X-barra es un modelo que permite estructurar construcciones gramaticales en tres niveles, permitiendo identificar relaciones entre los componentes de la estructura. Este modelo pertenece a la teoría de Principios y Parámetros, y fue 
Las acciones de modificación en una muestra de cuentos quechua del sur del Pastaza Alejandra Antoinette Dioses Tarazona

desarrollada por Ray Jackendoff. Sobre ello, Mioto, Figuereidos y Vasconcellos (2005) mencionan lo siguiente:

La teoría X-barra es el módulo de la gramática que permite representar un constituyente. Es necesaria para explicitar la naturaleza del constituyente, las relaciones que se establecen dentro de él y el modo como los constituyentes se ordenan jerárquicamente para formar una oración. Como sucede con cualquier módulo de la gramática, la teoría X-barra debe ser universal al punto de configurarse como un esquema general capaz de aprehender la estructura interna de los sintagmas de cualquier lengua. Pero también debe servir para dar cuenta de la variación entre las diferentes lenguas. (p. 5$)^{3}$

Bosque y Gutierrez (2008) explican que los sintagmas se articulan a un núcleo y este será el que les dé su nombre. Estos núcleos forman proyecciones sucesivas y establecen relaciones donde aceptan especificadores, complementos y adjuntos. Ellos también explican la teoría X-barra mostrando una estructura y mencionando lo siguiente:

La ESTRATEgia REDUCTORA a la que nos referimos tiene otras muchas consecuencias. Se aborda en la historia de la gramática generativa en Chomsky (1970) y sus hitos fundamentales son Jackendoff (1977) y Stowell (1981). Consiste, como vemos, en suponer que todas las proyecciones sintácticas, esto es, los sintagmas, están constituidas de manera similar. (p. 141)

Figura 1. Estructura sintagmática representada bajo la teoría $x$-barra.

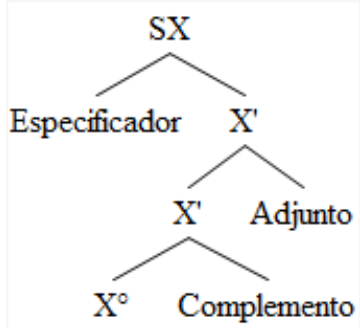

Nota: Tomado de Bosque y Gutierrez, 2008, p. 141

3 Cita extraída de la traducción realizada por Marisa Malcuori y Virginia Bertolotti del capítulo II del libro Novo Manual de Sintaxe.

120 Lengua y Sociedad. Revista de Lingüística Teórica y Aplicada 
En base a todo lo mencionado, y debido a que se analizan oraciones extraídas de cuentos que representan acciones, este trabajo busca identificar los sufijos de la variedad quechua del sur del Pastaza que señalen las funciones que cumplen las palabras dentro de la estructura predicativa de la acción, planteando, además, una distribución ordenada de estos elementos dentro de la estructura del predicado mediante gráficos arbóreos realizados bajo la teoría X-barra. Para ello, se cuestiona la existencia de sufijos específicos dentro de esta variedad quechua que denoten agentividad y atribucionalidad. Ante ello, se postula que existen sufijos específicos que denotan una entidad que cumple el rol de agente, una entidad paciente que sufre una modificación y una cualidad que será modificada, todo ello dentro de una acción.

\section{Metodología}

El estudio realizado es del tipo descriptivo no experimental, debido a que se busca identificar ciertos sufijos y sus funciones sin interferir sobre la muestra. La muestra de estudio está contenida en un libro llamado Cuentos folklóricos de los quechua del Pastaza, el cual recopila distintas narraciones de esta variedad quechua. A partir de este libro, se eligieron tres cuentos pertenecientes al primer apartado, que está denominado Animalpura kamanakurkanu (Sobre competencias y engaños entre animales). Los tres cuentos seleccionados fueron Karachupa pumamanta (El armadillo y el tigre), Karachupa kashka piwaniru (El armadillo flautista) y kunikitu mutiluwa (Un conejo y un motelo). De estos tres cuentos, se extrajeron quince oraciones como muestra para poder ser analizadas.

\section{Análisis}

El análisis de la muestra consiste, en primer lugar, en una segmentación morfológica mediante la cual se pueda evidenciar la función que cumplen los sufijos al modificar los lexemas. Además, se aprovechará la traducción al español que incluye el libro, la cual facilita la comprensión de los términos en esta variedad quechua, pero se empleará una traducción propia. A partir de ello, se formalizarán las oraciones para poder identificar la relación entre los elementos de las acciones de modificación. Finalmente, se elaborará una estructura arbórea del predicado de la acción mediante la teoría X-barra, donde se identifiquen a los argumentos con los roles que cumplen dentro de las acciones de modificación. 


\subsection{Datos}

En esta sección, se mostrarán las oraciones extraídas como muestra, su segmentación morfológica, la función de cada sufijo y la traducción de la misma oración. También se presentarán las formalizaciones de las acciones y su respectiva clase de acuerdo a la modificación que ocurre en dicha expresión. Además, se mostrarán dos gráficos por cada acción. El primero representará la acción en sí, donde se puede apreciar la estructura del predicado por cada verbo, señalando al agente y al paciente. El segundo representará la acción en términos de proceso y estado, es decir que mostrará uno de los estados del proceso que ocurre en la acción.

(1) Chaymantashi pumaka allarka uchkuta. Chaymanta-shi puma-ka alla-rka uchku-ta. Adv-Rep. Puma-Ag. Cavar-3Spas. Hueco-Acus. Entonces el puma cavó un hueco. $\aleph$ (pumaka, $\left[\neg \sum\right.$ (uchkuta, allashka) $\rightarrow \sum$ (uchkuta, allashka) $\left.]\right)$ - Dotación

Figura 2. Representación simple del predicado de allarka mediante X-barra.

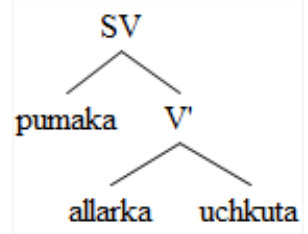

Figura 3. Representación de la acción en términos de proceso y estado.

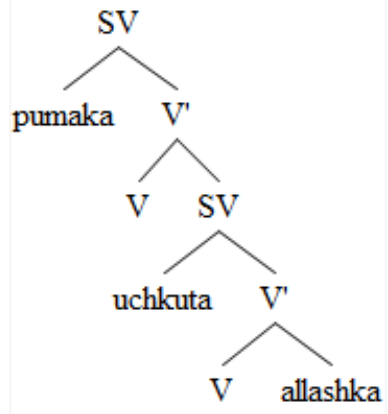


(2) Chaymantashi pumaka karachupapa chupanmantashi apirka.

Chaymanta-shi puma-ka karachupa-pa chupa-n-manta-shi api-rka. Adv-Rep. Puma-Ag. Armadillo-Gen. Cola-Pos3S-Abl-Rep.

Coger-3Spas.

Entonces el puma cogió la cola del armadillo.

$\aleph$ (pumaka, $\left[\neg \sum\right.$ (karachupapa chupanta, apishka) $\rightarrow \sum$ (karachupapa chupanta, apishka)]) - Dotación

Figura 4. Representación simple del predicado de apirka mediante X-barra.

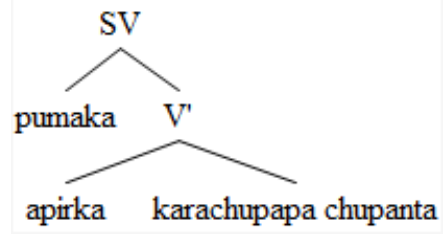

Figura 5. Representación de la acción en términos de proceso y estado.

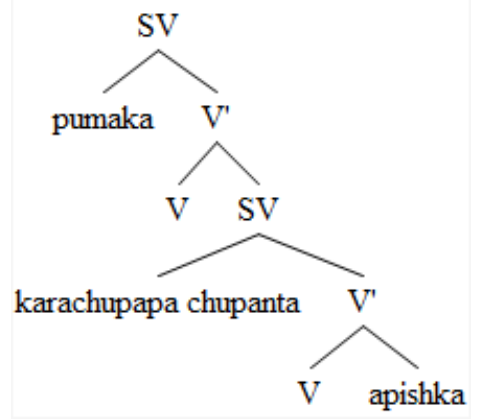

(3) Chayta uyashpanshi pumaka kacharirka.

Chay-ta uya-shpa-n-shi puma-ka kachari-rka.

Ese-Acus. Oír-Sub-3S-Rep. Puma-Ag. Liberar-3Spas.

Al escuchar eso, el puma lo liberó (al armadillo).

$\aleph$ (pumaka, $\left[\neg \sum\right.$ (karachupata, kacharishka) $\rightarrow \sum$ (karachupata, kacharishka)]) - Dotación 
(4) Chaypishi sirarka pumapa shiminta

Chaypi-shi sira-rka puma-pa shimi-n-ta.

Adv-Rep. Coser-3Spas. Puma-Gen. Boca-Pos3S-Acus.

Entonces (el armadillo) cosió la boca del puma.

$\aleph$ (karachupaka, $\left[\neg \sum\right.$ (pumapa shiminta, sirashka) $\rightarrow \sum$ (pumapa shiminta, sirashka)]) - Dotación

Figura 6. Representación simple del predicado de sirarka mediante X-barra.

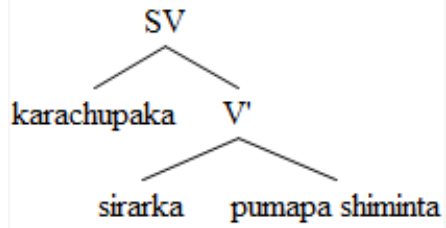

Figura 7. Representación de la acción en términos de proceso y estado.

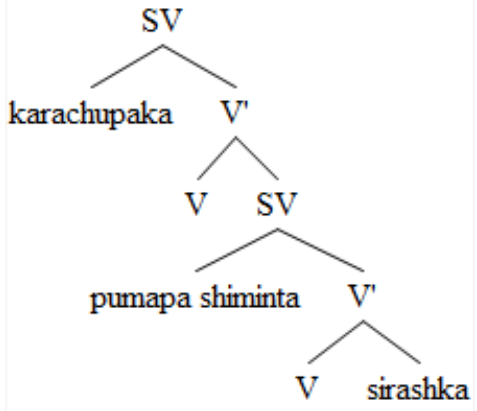

(5) Chaypishi pumaka apishpa mikurka karachupata.

Chaypi-shi puma-ka api-shpa miku-rka karachupa-ta.

Adv-Rep. Puma-Ag. Agarrar-Sub. Comer-3Spas. Armadillo-Ac.

Entonces, el puma agarró al armadillo y se lo comió.

$\aleph$ (pumaka, $\left[\neg \sum\right.$ (karachupata, apishka) $\rightarrow \sum$ (karachupata, apishka) $\left.]\right)$

- Dotación

$\aleph\left(\right.$ pumaka, [ $\sum$ (karachupata, kawsa) $\rightarrow \sum$ (karachupata, mikushka)])

- Alteración

124 Lengua y Sociedad. Revista de Lingüística Teórica y Aplicada 
Figura 8. Representación simple del predicado de mikurka mediante X-barra.

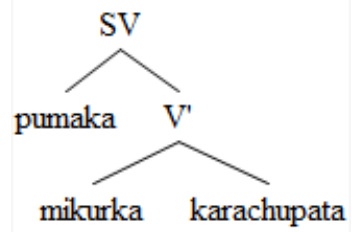

Figura 9. Representación de la acción en términos de proceso y estado.

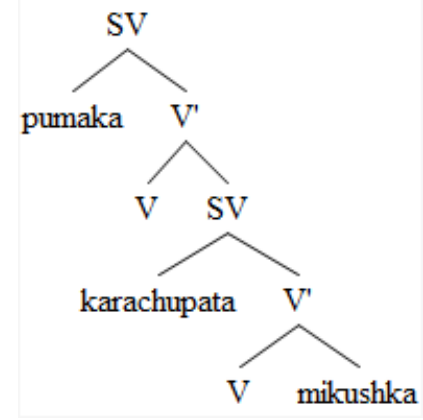

(6) Chaypi sirarka

pumapa shiminta.

Chaypi sira-rka puma-pa shimi-n-ta.

Adv. Coser-3Spas. Puma-Gen. Boca-Pos3S-Acus.

Entonces (el armadillo) cosió la boca del puma.

$\aleph$ (karachupaka, $\left[\neg \sum\right.$ (pumapa shiminta, sirashka) $\rightarrow \sum$ (pumapa shiminta, sirashka)]) - Dotación

(7) Pumapa shiminta sirarka karachupaka.

Puma-pa shimi-n-ta sira-rka karachupa-ka.

Puma-Gen. Boca-Pos3S-Acus. Coser-3Spas. Armadillo-Ag.

El armadillo cosió la boca del puma.

$\aleph$ (karachupaka, $\left[\neg \sum\right.$ (pumapa shiminta, sirashka) $\rightarrow \sum$ (pumapa shiminta, sirashka)]) - Dotación

(8) Apipayarka chupanmanta.

Api-paya-rka chupa-n-manta.

Agarrar-Hab-3Spas. Cola-Pos3S-Ablat.

(El puma) Lo agarró (al armadillo) de su cola.

$\aleph$ (pumaka, $\left[\neg \sum\right.$ (karachupapa chupanta, apishka) $\rightarrow \sum$ (karachupapa chupanta, apishka)]) - Dotación 
(9) Chayrayku sakirka pumaka.

Chayrayku saki-rka puma-ka.

Por eso Liberar-3Spas. Puma-Ag.

Por eso el puma lo liberó (al armadillo).

$\aleph$ (pumaka, $\left[\neg \sum\right.$ (karachupata, sakishka) $\rightarrow \sum$ (karachupata, sakishka)]) - Dotación

(10) Chaypi sakmarka makinka apirirka chaypi.

Chaypi sakma-rka maki-n-ka apiri-rka chaypi.

Adv. Abofetear-3Spas. Mano-Pos3S-Ag. Pegarse-3Spas. Adv.

Entonces lo golpeó con su mano y se pegó allí.

$\aleph$ (makinka, $\left[\neg \sum\right.$ (runata punkaramanta, sakmashka) $\rightarrow \sum$ (runata punkaramanta, sakmashka)]) - Dotación

Figura 10. Representación simple del predicado de sakmarka mediante X-barra.

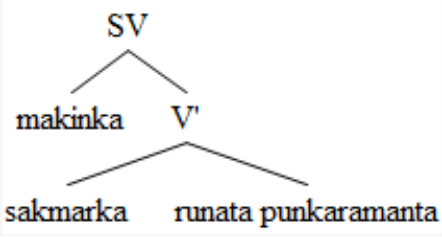

Figura 11. Representación de la acción en términos de proceso y estado.

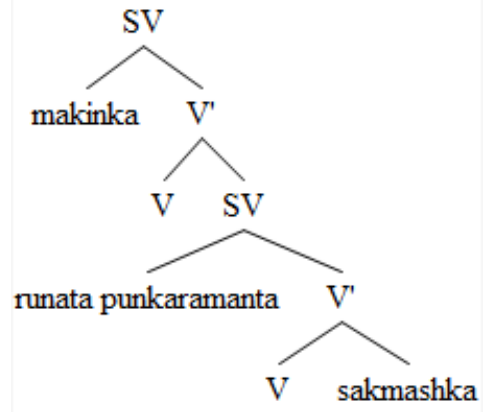

(11) Kuti shuk makinwa waktarka, apirirka.

Kuti shuk maki-n-wa wakta-rka apiri-rka.

Otra vez Mano-Pos3S-Instr. Golpear-3Spas. Pegarse-3Spas.

Otra vez, (el conejo) golpeó con su mano, se pegó.

$\aleph$ (makinka, $\left[\neg \sum\right.$ (runata punkaramanta, waktashka) $\rightarrow \sum$ (runata punkaramanta, waktashka)]) - Dotación 
(12) Patiyarka, chaypi apirirka.

Patiya-rka, chaypi apiri-rka.

Patear-3Spas. Adv. Pegarse-3Spas.

(El conejo) Lo pateó (al muñeco), allí se pegó.

$\aleph$ (kunkituka, $\left[\neg \sum\right.$ (runata punkaramanta, patiyashka) $\rightarrow \sum$ (runata punkaramanta, patiyashka)]) - Dotación

Figura 12. Representación simple del predicado de patiyarka mediante X-barra.

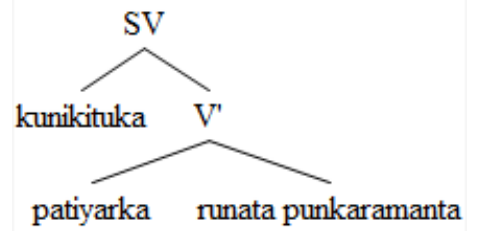

Figura 13. Representación de la acción en términos de proceso y estado.

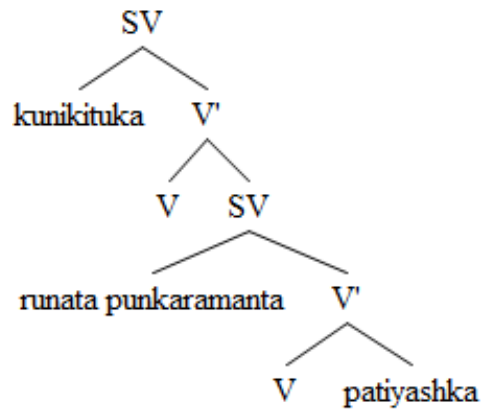

(13) Patiyashka shuk chakinwa, llampu chakinka apirirka. Patiya-shka shuk chaki-n-wa, llampu chaki-n-ka apiri-rka. Patear-3Spas. otro pie-Pos3S-Instr. entero pie-3SPos-Ag. Pegarse-3Spas. Pateó con el otro pie, su pie entero se pegó.

$\aleph$ (kunkituka, $\left[\neg \sum\right.$ (runata punkaramanta, patiyashka) $\rightarrow \sum$ (runata punkaramanta, patiyashka)]) - Dotación 
Las acciones de modificación en una muestra de cuentos quechua del sur del Pastaza Alejandra Antoinette Dioses Tarazona

(14) Chaypi watarka pumata kunikituka.

Chaypi wata-rka puma-ta kunikitu-ka.

Adv. Atar-3Spas. Puma-Acus. Conejo-Ag.

Allí el conejo ató al puma.

$\aleph$ (kunikituka, $\left[\neg \sum\right.$ (pumata, watashka) $\rightarrow \sum$ (pumata, watashka) $\left.]\right)$

- Dotación

Figura 14. Representación simple del predicado de watarka mediante X-barra.

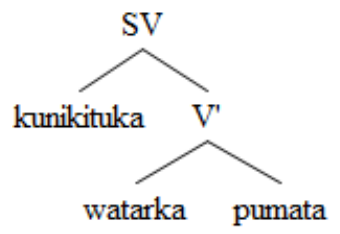

Figura 15. Representación de la acción en términos de proceso y estado.

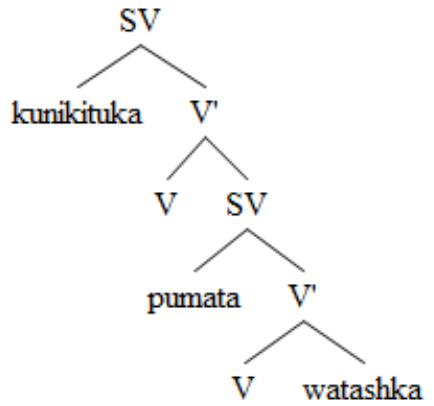

(15) Chaypi wañuchirka chay pumata.

Chaypi wañu-chi-rka chay puma-ta.

Adv. Morir-Caus-3Spas. Ese Puma-Acus.

Entonces (el motelo) mató al puma.

$\aleph$ (mutiluka, [ $\sum$ (pumata, kawsa) $\rightarrow \neg \sum$ (pumata, kawsa)]) - Detracción 
Figura 16. Representación simple del predicado de wañuchirka mediante X-barra.

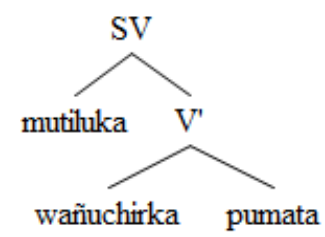

Figura 17. Representación de la acción en términos de proceso y estado.

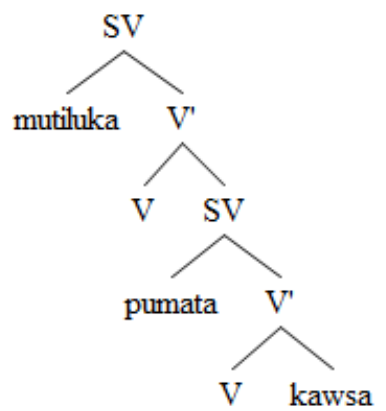

A continuación, se presenta una tabla que contiene palabras y sufijos pertenecientes al léxico quechua de la variedad del sur del Pastaza, las cuales forman parte de los datos, donde se muestran sus funciones y significados para un mayor entendimiento.

Tabla 3. Léxico quechua del sur del Pastaza empleado en el análisis del corpus.

\begin{tabular}{|l|l|}
\hline Léxico quechua & Función-significado \\
\hline Chaymanta & Adverbio (Adv). Entonces \\
\hline -shi & Reportativo (Rep). \\
\hline -ka & Agentivo (Ag). \\
\hline -rka/-shka & Sufijo de tiempo pasado en $3^{\circ}$ p. singular \\
\hline -shka & $\begin{array}{l}\text { Adjetivo participio. Estado que resulta } \\
\text { luego de ser afectado por el verbo. }\end{array}$ \\
\hline -ta & Caso acusativo, od (Acus). \\
\hline -pa & Caso genitivo (Gen). \\
\hline -manta & Caso Ablativo (Abl). De. \\
\hline -n & $\begin{array}{l}\text { Sufijo posesivo } 3^{\circ} \text { p. singular. Sufijo de } \\
\text { acción de } 3^{\circ} \text { p. singular. }\end{array}$ \\
\hline
\end{tabular}


Las acciones de modificación en una muestra de cuentos quechua del sur del Pastaza Alejandra Antoinette Dioses Tarazona

\begin{tabular}{|l|l|}
\hline Léxico quechua & Función-significado \\
\hline Chaypi & Adverbio (Adv). Allí. Entonces \\
\hline -ri & Reflexivo (Reflex). \\
\hline -shpa & Subordinante (Sub). \\
\hline -hu & Progresivo (Prog). \\
\hline -ra & Acción simultánea (Sim). Todavía. \\
\hline -paya & $\begin{array}{l}\text { Indica aspecto habitual en tiempo } \\
\text { pasado y presente (Hab). }\end{array}$ \\
\hline Ali, alita. & Adj. bien. Adv. bueno. \\
\hline Kawsa & Adj. vivo. \\
\hline Chayrayku & Conjunción. Por eso. \\
\hline $\begin{array}{l}\text { Runa } \\
\text { punkaramanta }\end{array}$ & Hombre de brea. \\
\hline -chi & Causativo (Caus). \\
\hline
\end{tabular}

\subsection{Resultados}

Debido a que las oraciones analizadas están dentro de un texto narrativo, los agentes y/o pacientes no estaban especificados gráficamente en algunos casos, es decir, se omitieron, puesto que se puede sobreentender por el contexto.

En la mayoría de oraciones se observó al agente marcado con el sufijo $-k a$, sin embargo, este agente no aparecía en todas las oraciones de manera explícita, es decir, era tácito. Respecto a la identificación de la entidad paciente, se notó que esta llevaba la marca del caso acusativo en la mayoría de casos, la cual se expresa mediante el sufijo - $t a$, sin embargo, en los casos de las oraciones 2 y 8 , donde la acción refiere a agarrar una parte del animal (en este caso la cola), el sufijo acompañante era el de caso ablativo -manta, interpretándose como lo agarró por la cola y no como agarró la cola, es por esa razón que en las formalizaciones y en las estructuras arbóreas se les ha identificado a las entidades pacientes con el sufijo -ta. En cuanto a las propiedades atributivas, se empleó el sufijo -shka dentro de las formalizaciones y las estructuras arbóreas para señalarlas cuando correspondían a verbos transformados en adjetivos participios (comer: comido, agarrar: agarrado, etc.), pero también se utilizó algunas glosas identificadas naturalmente como adjetivas sin añadirles sufijo alguno. A continuación, se presenta una tabla resumiendo lo mencionado anteriormente, donde se indica el rol dentro de la acción que señalaría cada sufijo. 
Tabla 4. Sufijos quechua empleados para indicar los roles de la acción en la variedad del sur del Pastaza.

\begin{tabular}{|l|l|}
\hline Rol & Sufijo \\
\hline Agente & -ka \\
\hline Paciente & -ta, -manta \\
\hline Atribución & -shka* \\
\hline
\end{tabular}

El análisis de las oraciones mediante la teoría X-barra ha sido simple debido a que solo se buscó identificar los papeles semánticos dentro de las acciones, es por ese motivo que se obviaron ciertos elementos como algunos adverbios y conjunciones. Además, se han analizado oraciones similares que poseen la misma formalización, sin embargo, algunas poseen la partícula del reportativo -shi, lo cual nos indicaría cierta veracidad si son analizadas desde la Teoría de modelos, generando que todas las oraciones sean diferentes de acuerdo a sus valores de verdad.

En el análisis de la oración 5, se consideró al estado inicial con la cualidad de vivo y no de no comido porque es el estado más natural. Sin embargo, a pesar de que al estar comido también está muerto, no se consideró como una detracción, sino como una alteración puesto que la entidad cambiaría no solo su estado sino que también transformaría su forma.

\section{Conclusiones}

A partir de lo examinado en el presente trabajo, podemos afirmar que existen sufijos específicos en la variedad quechua del sur del Pastaza que denotan algunos de los papeles semánticos que constituyen las acciones, entre ellos, - $k a$ para señalar el rol de agente y - ta para señalar el rol de paciente. Respecto a este último participante, se identificó que en algunos casos iba acompañado del sufijo -manta, pero solo aparece cuando se hace referencia a determinada parte de la entidad y no el individuo por completo. En cuanto a la denotación de la propiedad atributiva dentro de las acciones, no ha sido posible considerar un sufijo específico que cumpla con dicha función puesto que dependerá de la categoría gramatical de la palabra. Por esa razón, el sufijo -shka ha sido utilizado para construir participios que puedan cumplir con el rol de la atribución en los casos donde no se ha podido encontrar un término adecuado, para lo cual se empleó el Diccionario Inga Castellano. 
Otra de las partículas morfológicas encontradas en los relatos ha sido el sufijo -shi, el cual es un marcador de evidencialidad de segunda mano. Este sufijo no se ha presentado en todos los enunciados, sin embargo, ha servido para aseverar que lo descrito en dichas oraciones posee un rasgo de veracidad a pesar de que las narraciones describen situaciones ficticias donde los personajes involucrados son animales que, en algunos casos, realizan acciones humanas. Además, se pudo identificar que no había la necesidad de mencionar explícitamente a las entidades que cumplían los roles de agente y paciente en algunos enunciados debido a que se les podía identificar mediante algunos referentes textuales y la sufijación verbal.

Finalmente, se considera que se debe continuar realizando más estudios a profundidad bajo el enfoque de la Semántica formal en esta variedad quechua debido a que son escasos los trabajos de este tipo y así contribuir con la investigación y descripción de más aspectos lingüísticos correspondientes a esta lengua.

\section{Agradecimientos}

Se agradecen los alcances proporcionados por la lingüista Verónica Lazo, profesora del curso de Semántica in en la Universidad Nacional Mayor de San Marcos, quien brindó observaciones y revisiones para la confección del presente manuscrito.

Financiamiento

La investigación se realizó sin financiamiento.

\section{Conflicto de intereses}

Los autores no presentan conflicto de interés. 


\section{Referencias bibliográficas}

Bosque, I. y Gutierrez, J. (2008). Fundamentos de sintaxis formal. Akal. Ethnologue. Quechua, Southern Pastaza. https://bit.ly/2xyomCo

Guerrero, G. (2010). La noción de modelo en el enfoque semántico de las teorías. Praxis Filosófica, (31), 169-185. https://bit.ly/3Esadou

Instituto Lingüístico de Verano. Quechua del sur de Pastaza. https://bit.ly/3Ep4JD1 Mioto, C., Figuereido, M. y Vasconcellos, R. (2005). Novo Manual de Sintaxe (Marisa Malcuori y Virginia Bertolotti, Trad.). Florianópolis: Editora Insular. ( $2^{\text {a }}$ ed.). Capítulo II. (Obra original publicada en 2005)

Moreno, C. (2003). Semántica y Gramática. Sucesos, papeles semánticos y relaciones sintácticas. Machado.

Muñoz, C. (2006). Semántica Cognitiva: Modelos Cognitivos y Espacios Mentales. A Parte Rei, 43, 01-28. https://bit.ly/3CpG7JG

Santos, L. (2017). El dogma extensionalista de las aproximaciones veritativo-condicionales del significado. Revista Internacional de Filosofía, 22 (2), 155-172. https://bit.ly/3mrB59U

Tödter, C., Waters, A. y Zahn, C. (1998). Cuentos folklóricos de los quechua del Pastaza. Instituto Lingüístico de Verano.

Vilcapoma, J. (2016). Las características culturales de los grupos etnolinguísticos quechuas del Pastaza. Anales Científicos, 77 (2), 290-297. https://bit.ly/3blcwoP

Wise, M.(Ed.). (2002). Shimikunata asirtachikkillka Inka Castellanu. Diccionario Inga $\sim$ Castellano. Instituto Lingüístico de Verano.

\section{Trayectoria académica}

Alejandra Antoinette Dioses Tarazona, estudiante de Lingüística en la Universidad Nacional Mayor de San Marcos, con interés en estudios de andinística, semántica y sociolingüística. 